\title{
Philosophiques
}

\section{Expérience, communauté, institution. Réflexions sur la spécialisation de l'éducation morale au primaire}

\section{Georges Leroux}

Volume 14, numéro 2, automne 1987

URI : https://id.erudit.org/iderudit/027020ar

DOI : https://doi.org/10.7202/027020ar

Aller au sommaire du numéro

Éditeur(s)

Société de philosophie du Québec

ISSN

0316-2923 (imprimé)

1492-1391 (numérique)

Découvrir la revue

Citer ce document

Leroux, G. (1987). Expérience, communauté, institution. Réflexions sur la spécialisation de l'éducation morale au primaire. Philosophiques, 14(2),

413-423. https://doi.org/10.7202/027020ar d'utilisation que vous pouvez consulter en ligne. 


\title{
INTERVENTION
}

\section{EXPÉRIENCE, COMMUNAUTÉ, INSTITUTION. RÉFLEXIONS SUR LA SPÉCIALISATION DE L'ÉDUCATION MORALE AU PRIMAIRE}

\author{
par Georges Leroux
}

\begin{abstract}
Dans la communication que je propose à votre réflexion, se trouvent joints deux éléments que je discute comme s'il était possible d'en traiter de manière clairement séparée : l'expérience, d'une part et d'autre part l'institution. La critique très générale à laquelle je recours pour tenter de restituer au concept d'expérience la profondeur qu'il me semble avoir perdue au profit de celui d'habileté et de technique, n'a cependant pour terrain d'application que la seule institution scolaire de notre milieu, et je restreindrai mon exposé à l'école primaire. Ma question pourrait se résumer de la manière suivante : à quel concept d'expérience nous renvoie la tendance actuelle à spécialiser les savoirs, et notamment à spécialiser l'éducation (ou la formation) morale? Un certain nombre de considérations historiques seront requises pour poser correctement cette question, mais j'essaierai de garder à mon questionnement une ambition philosophique. Je le place d'emblée sous l'égide de la figure de Dewey, auquel j'essaierai en terminant, d'apporter un hommage inattendu en même temps que j'y trouve le modèle d'une pratique espérée.
\end{abstract}

Je commencerai par tenter de formuler clairement la question que pose à la philosophie le phénomène qui nous rassemble ici. $\mathrm{Ce}$ 
phénomène est celui de la substitution à un magistère religieux spécialisé, d'une autorité qui emprunte sa légitimité et sa conceptualité à la philosophie elle-même, à la morale et à la logique. Nous assistons en effet au phénomène d'une culture qui, saisie par le reflux des rôles spécialisés du magistère religieux, ne trouve rien de mieux à faire que d'en répéter les fonctions. L'ancienne culture, pénétrée d'une autorité extérieure à elle-même, en même temps que pleinement intériorisée, avait produit une institution scolaire où l'enseignement religieux constituait, à toutes fins utiles, la seule spécialisation. Le magistère de l'Église, fortement hiérarchisé, ne pouvait en effet s'accommoder de ce que les simples instituteurs assument par eux-mêmes la transmission des enseignements catéchétiques; partout donc où les ressources le permettaient, c'était l'aumônier de l'école qui remplissait cette fonction, ou à tout le moins un maitre que sanctionnait son statut religieux. Cette forme d'institution a créé un rôle qui liait fortement l'école à la communauté sociale, dans la préparation et la participation rituelle aux sacrements. On n'insistera jamais assez sur la force de cette unité organique, dont les aspects didactiques assurés par l'école avaient d'abord une fin plus haute et surtout plus large, savoir la ritualisation de la communauté.

Pour des raisons qu'il ne m'appartient pas d'expliquer, non seulement cette ritualisation s'est effritée, principalement sous l'influence d'une érosion de la croyance, mais encore n'a-t-elle pas été remplacée. Aucune articulation de la communauté ne s'est substituée à celle qu'avait produite la culture ancienne, et qui portait organiquement l'enfant, enchâssé dans une formation doctrinale, à la connaissance et à la pratique de la vie sacramentaire. En laissant se dissoudre, ce lien à la fois cognitif, - corrélé à la maîtrise d'une vision métaphysique du monde - et pratique, c'està-dire effectué dans un rapport senti aux obligations et aux devoirs de la communauté, notre culture a laissé béants des espaces sociaux que ne pourrait remplir qu'une communauté nouvelle.

Celle-ci n'est pas advenue. Partout et de toutes manières, elle manque, jusque dans son point de jonction archaïque, qui était par ailleurs le pivot de la moralisation de l'enfant, la famille biparentale traditionnelle.

La disjonction de la communauté et de la structure sociale substitue à une articulation réglée par des rituels et une vision 
organique de la vie une juxtaposition de rôles et de tâches dépourvues de finalités. La mort de Dieu et de tout sacré a rendu vide de sens toute autre forme de vie que cette juxtaposition sans linéarité et sans principe de l'expérience.

Il aurait été paradoxal que l'école ne manifestât pas les conséquences de cette désarticulation du rituel et de ses justifications fondatrices. Dans une culture aliénée, l'école est naturellement l'institution où l'aliénation de l'expérience est la plus vivement ressentie, à proportion qu'elle a destin d'en transmettre non seulement les savoirs accumulés, mais les principes et l'énergie. L'école publique, naturellement ouverte à ce qu'on appelle le pluralisme, qui n'est qu'un autre nom pour la fragmentation dont je parle, n'a pas encore commencé d'en faire la théorie. Mais cela ne l'empêche pas de croire, - et il faut y voir un réflexe de vie que nonobstant la désarticulation dont elle subit les effets jusque dans la désintégration des familles, elle peut être encore, elle, une communauté.

C'est cette raison, et il me semble falloir y attacher une importance extrême, qui explique que contre toutes les forces militant en sens contraire, l'école veuille s'investir d'une définition organique que rien dans le tissu social tel qu'il existe actuellement ne lui permet de penser réaliser. En effet, l'école travaille contre la désarticulation et ses effets d'indifférence et de détéléologisation de l'expérience, elle incorpore une préoccupation morale qui la définit comme communauté ; elle se crée comme communauté, là où tout l'exige, mais rien ne le permet.

Dans le mouvement particulier de technicisation des savoirs, de spécialisation des apprentissages, il se peut cependant que l'école, subissant le pli porté par l'ancienne culture, fasse actuellement les mauvais choix. Je reviens à mon questionnement du point de départ. Si ma description est juste, on aurait pu attendre que ce travail en vue de la communauté ne s'effectue pas en renforçant le mouvement de la spécialisation, mais en quelque sorte en travers de lui. Étrangement, c'est au contraire qu'on assiste et ce phénomène me semble tout à fait inquiétant.

L'absence de communauté réelle, la dissolution dans une urbanité que ne compense aucune famille, ni grande ni petite, a laissé ouverte, béante la place du magistère. De la préparation à la 
communauté sacramentaire, on passe sans transition à l'expertise morale ; la place n'est pas restée longtemps inoccupée, la communauté ne s'est pas donné le temps d'éprouver l'abîme des valeurs sur lequel elle se trouve suspendue. L'ensemble des tendances et des programmes qui en découlent, représente une concurrence remarquable pour occuper la place du magistère : clarification des valeurs, philosophie pour enfants, approches développementales kohlbergiennes. Malgré leurs différences profondes sur le plan des approches, tous ces efforts sont animés par une volonté commune, qui est d'investir l'école d'une représentation de la communauté.

Je tiens à clarifier d'emblée ma position à ce stade de l'exposé; je crois que cet énoncé de l'objectif est absolument acceptable, et même sans doute est-ce le seul possible aujourd'hui. Sa nécessité n'est ni plus grande ni moins grande que celle des structures archaïques qui liaient le mythe et le rite, et la production d'une organicité consensuelle susceptible de répondre aux modèles scientifiques du monde peut être considérée comme une tâche isomorphe à celle qui produisit l'équilibre traditionnel représenté extraordinairement, par exemple, dans le rapport de la liturgie au calendrier agraire.

Un équilibre nouveau, encore à venir et sans qu'on puisse en voir encore même l'esquisse, ne pourra se produire, a priori, que dans une communauté d'apprentissage. Cette question était déjà, chez Dewey, motivée politiquement par l'exercice de la démocratie, mais l'exemple actuel de notre culture lui donne un relief plus étendu; c'est la totalité de l'expérience morale de l'humanité, et pas seulement ses formes politiques évoluées, qui cherche les assises de sa continuation.

La question est cependant la suivante: l'expérience morale répond-elle d'un savoir dont la spécificité, en général confirmée par ses traits de formalité par opposition à des savoirs de contenus, engage à un apprentissage spécialisé ? Est-ce le leurre de la fonction propre au magistère de la culture ancienne qui nous fait tendre à occuper sans poser plus de questions la place laissée vacante par le catéchète? 


\section{II}

Cette réflexion sur le destin de l'institution comme représentant symbolique de la communauté nous permet de revenir à la question de l'expérience. La culture religieuse occidentale s'était substituée à la culture gréco-romaine, marquée sans doute par une forte imprégnation du politique; mais comme cela se voit autant chez Aristote que dans la tradition augustinienne des deux cités, la morale était toujours d'emblée déterminée par une instance supérieure : politique, théologie. La sécularisation, qui fait le fond de la légitimité de la modernité selon l'expression de Blumemberg, a eu un effet de renforcement de l'importance de la morale que l'ancien monde n'a pas connue. La morale est le fond résistant de l'expérience quand le politique et le théologique ont laissé s'effacer la figure de leur domination.

L'érosion des fondations, la sédimentation des idéologies, loin d'aplatir la revendication morale de l'individu, contribuent au contraire à renforcer les idéaux de liberté, d'autonomie, de justice. La question devient rapidement celle de savoir comment alléguer ces idéaux en l'absence d'une communauté, théologiquement ou politiquement fondée?

Si donc l'institution scolaire, substitut de tous les substituts, complément de tous les compléments, accélère la fragmentation des tâches et des rôles, ne se privera-t-elle pas elle-même d'un engagement dans la voie de la communauté que tout requiert d'elle dans le moment? Cette question exige que soit précisé le rapport entre l'idée de communauté et la moralisation de l'expérience. Je laisse d'abord de côté, l'idée pourtant répandue, selon laquelle la famille est le noyau de toute représentation de la communauté, noyau sur lequel la société peut se replier pour la moralisation de l'expérience. Cette idée me semble contredite par les faits. La famille n'est pas seulement désintégrée; même dans le cas de groupes unis, elle est envahie par une représentation sociale médiatique qui lui interdit de se suffire. Je laisse aussi de côté les utopies communautaires des années soixante-dix ; ces utopies ont produit une extraordinaire révélation de la nécessité et du besoin de la moralisation de l'expérience par la communauté, mais elles n'ont pas réussi à rencontrer les exigences de l'ère post-industrielle, de la massification, de la technologie, etc... 
Je m'adresse donc à ce premier niveau de l'urbanité postindustrielle où le projet de communauté est encore ressaisi par ceux qui l'habitent, essentiellement les enseignants et les parents intéressés, y compris les comités d'école.

Dans cette institution, qui se souvient encore des principes de son organicité passée, le passage à une juxtaposition de services semble inévitable. On apprendra aux enfants la langue, et à côté de cela les mathématiques, et à côté encore la musique, et à côté encore la moralité critique.

Chacun sait pourtant combien ce modèle de l'articulation des activités n'est pas celui qu'il revendique, et chacun veut investir une représentation de la communauté où chaque apprentissage est subordonné à un centre qui commande toute la représentation. La langue et les savoirs, comme la moralité, n'ont de sens que dans leur rapport à cette communauté, dont la figure paternelle, archaïque a disparu. L'école, malgré toute sa volonté, ne peut remplacer ce dieu perdu. C'est pourtant ce qui lui est demandé : elle remplace la famille, intercède dans le vacuum créé par la séparation du père et de la mère; plus encore, elle substitue au rythme archaïque du temps saisonnier un calendrier arbitraire, en proposant des ritualisations des anciennes fêtes maintenant dépourvues de leur mythe.

Malgré qu'elle n'ait aucune raison de croire en posséder la légitimité, l'institution s'adonne à cette fiction comme à une nécessité vitale. Un tel investissement n'a pas besoin d'être jugé, il n'a qu'à être constaté et cette constatation rencontre sur son chemin, très rapidement, le lien entre la moralisation de l'expérience et la volonté de communauté. Là où l'école traditionnelle n'était qu'une institution de relais, la voici en passe de devenir la seule communauté réelle : l'obligation d'être ensemble pour apprendre, pour se développer, quelles qu'en soient les finalités, et quels que soient les rites destinés à en émerger. Cette communauté réelle ne peut survivre que si son expérience est moralisée.

La question est de savoir si elle doit l'être comme une habileté spécifique, ou si elle doit l'être dans son projet d'ensemble. Moraliser l'expérience, pour faire court, c'est apprendre à y développer des habitus formels généralisables, capables de guider la décision, exercés dans une multitude d'activités, et renforcés notamment par des exercices spécifiques. Je ne discuterai pas ici la 
question des objets de l'expérience morale (la vie bonne, la sagesse, la vertu et le développement du caractère, le traitement rationnel de l'intersubjectivité, la justice, etc...) Tous ces objets, qui peuvent être considérés comme des finalités ultimes, sont suspendus à une idée de la communauté, qui, si elle vient à disparaître, entraîne leur désagrégation.

Mon hypothèse, à ce stade de notre réflexion, est qu'en l'absence d'une intégration à l'ensemble de l'expérience, le développement d'apprentissages spécifiques, de programmes experts, ne va contribuer qu'à éroder davantage l'idée de communauté et à rendre abstraites et intellectuelles les finalités ultimes de la vie éthique. Abstraites, parce que dégagées des investissements et des enjeux réels, quotidiens, de la communauté qui se joue dans l'institution scolaire primaire ; intellectuelles parce que transmises nécessairement sur le mode d'une expertise dégagée de l'expérience, par quelqu'un qui est sans rapport avec cette expérience, qui est incapable d'en mesurer les difficultés, et qui n'a accès, somme toute, qu'à ce à quoi sa compétence l'a préparé : la technicité d'un apprentissage finement découpé, mais sans rapport à la communauté. L'éducation dans sa totalité est une entreprise morale, dans la mesure où elle s'articule sur un projet de communauté : même si celle-ci est en éclats, même si l'école lutte pour faire croire à la communauté dans la fiction d'un pluralisme susceptible de parole et de communication, même si rien de cohérent ne peut être attendu d'aucune autre communauté pour le soutien ou le prolongement du projet...

L'éducation morale doit-elle faire l'objet d'une activité spécifique? Ma proposition est de réserver à l'école secondaire la compartimentation des apprentissages moraux, pour un moment de la vie où cette compartimentation n'attaque pas la représentation de la communauté, et de conserver au primaire un cadre intégré, où tous les objectifs développés actuellement par ceux qui ont souci de contribuer à la moralisation de l'expérience, n'auront pas un terrain d'application spécifique, mais une portée sur la totalité de l'expérience scolaire.

À cet effet, la préparation des intervenants ne devrait pas avoir pour finalité de produire des experts, mais de renforcer dans la formation de tous les maîtres, une compétence de moralisation/ 
/éducation morale destinée à enrichir la communauté que cherche à créer l'école primaire. À l'époque où cette compétence n'était pas requise, parce que l'organicité morale était garantie et soutenue par une articulation religieuse, cet investissement n'était pas nécessaire. Mais aujourd'hui, la communauté ne peut être confiée à la seule compartimentation des savoirs particuliers; elle ne peut tolérer d'homologuer l'éducation morale à une fonction de ce genre, elle doit au contraire travailler à recentrer l'expérience sur un principe d'organisation, pour contrer les forces centrifuges de la spécialisation.

Une telle proposition prend en compte la totalité de la tâche qui repose sur l'école primaire comme communauté ; elle prend au sérieux le fait que les enfants, les parents et les intervenants (professeurs animateurs de classes et professeurs spécialistes) forment une communauté complète, où la vie scolaire est l'équivalent de la vie sociale démocratique, comme aimait à le rappeler Dewey. Ce rapport symétrique de l'école et de la communauté politique induit une continuité dans l'expérience qui peut servir de principe au refus de la spécialisation à l'école primaire.

On peut en risquer la formulation suivante : toute l'expérience sociale, dans toutes ses dimensions, peut être étendue et mesurée à l'ensemble de la vie scolaire. Si nous cherchons, au-delà d'une détermination politique particulière comme celle de la démocratie à laquelle recourait Dewey, à décrire cette expérience sociale, nous serons mis en présence de constituants éthiques ultimes de la communauté : la reconnaissance d'une normativité, le respect de la subjectivité, l'idéal de la liberté, le droit à la différence, la nécessité de justifier et de rationaliser les choix, les fondements de la sanction et de la punition. Chaque moment de l'expérience qui permet de rencontrer, de faire face à un quelconque de ces constituants, est aussi un moment qui moralise l'expérience; et chaque moment éthique est un moment qui crée la communauté. Aucun ne saurait donc être restreint à l'apprentissage d'une fonction spécifique, par exemple par le moyen de fictions non reliées au développement de l'expérience.

Une argumentation plus développée pourrait sans doute montrer les effets néfastes d'une spécialisation sur la constitution même de l'idée de communauté comme fondement ultime de la vie 
éthique chez les enfants. La communauté même qui devrait leur fournir des normes leur est représentée comme une structure délabrée ; il leur appartient de la reconstruire, mais ils ne peuvent compter pour cela sur aucun des moyens normatifs que la génération antérieure a vu se désagréger si rapidement. Renvoyés à leur expérience, les enfants ne peuvent que créer un modèle de communauté et celui-ci n'aura d'intérêt que s'il est autre chose qu'un petit exercice de spécialistes. Aucun des constituants que j'ai mentionnés plus haut n'apparaît dans l'expérience avec la force d'un fondement ; qu'est-ce que l'idéal de liberté, en effet, dans une société systématiquement contrôlée par l'information médiatique ? Les effets dévastateurs de la télévision sur l'école, qu'elle contribue à homogénéiser par le bas, commencent à peine à être saisis par les enfants euxmêmes ; cet exemple, comme tant d'autres, indique comment la spécialisation travaille contre la constitution de la communauté. Toute l'expérience est touchée et elle doit être moralisée de part en part, c'est-à-dire confrontée d'une part à son délabrement, à son aliénation et d'autre part aux exigences éthiques de la communauté réelle de l'école.

\section{III}

Je voudrais, en terminant, saluer du point de vue qui a été le mien la figure immense de Dewey. Chacun sait ce que notre concept de l'expérience lui doit et comment une réflexion sur l'expérience modifie notre approche des apprentissages et des habiletés. En insistant sur un cadre intégré pour l'école, Dewey a fait voir comment l'éducation est une entreprise morale autant qu'intellectuelle. De la sorte, il a fourni, avant même que cela nous apparaisse comme un besoin impérieux, tout ce qu'il fallait pour contrer l'intellectualisme dont il ne pouvait prévoir l'extraordinaire développement, sous l'influence notamment de la concurrence des psychologies.

Déjà, dans son texte de 1897 intitulé Etbical Principles Underlying Education ', il confiait à l'école une responsabilité complète, sur tous les plans de l'expérience où celle-ci peut être

1. John DewEY, The Early Works, 1882-1898, vol. 5:1895-1898, Early Essays. Carbondale \& Edwardsville, Southern Illinois University Press, 1972, pp. 54-83. 
moralisée ; aucune limite ne peut être assignée à cette tâche, qui doit être interprétée de la manière la plus large et la plus libre. Pas seulement, et pas même principalement des apprentissages spécifiques, mais d'abord une prise de possession de soi-même qui branche l'enfant sur la vie sociale. Aucun apprentissage spécifique ne peut en effet être dégagé de la vie sociale à laquelle il doit contribuer "Nous ne découvrirons aucun idéal moral, aucune valeur morale pour la vie de l'école, sauf si nous leur donnons une interprétation sociale. ${ }^{2}$

C'est cet aspect du rapport à la réalité de la vie scolaire, comme communauté pratique existant par elle-même, qui rend futile l'intervention d'un spécialiste des valeurs ou du raisonnement critique, ou de quelque autre habileté; cette intervention n'a de sens que si elle est reliée à la totalité de l'expérience, saisie dans sa continuité, saisie dans sa réalité. L'accent mis par Dewey sur l'intégration de l'éducation morale et de tous les apprentissages dans les situations concrètes de la vie scolaire n'a fait que se renforcer. Quand nous le retrouvons dans Democracy and Education, qui date de $1916^{3}$, nous sommes confrontés à une définition de l'éducation comme intrinsèquement morale, en tant qu'elle est organisation et reconstruction constante de l'expérience. Cette idée devait, chez Dewey, connoter celle de la création continue de la signification morale, laquelle entraîne la possibilité d'une création continue de la communauté et des institutions.

En invoquant la figure de Dewey à la fin de cette réflexion, j'ai voulu montrer et en quelque sorte désamorcer le caractère par trop prévisible du développement des techniques de l'éducation morale à l'école primaire; n'est-il pas urgent de ressaisir le concept d'expérience légué par Dewey à toute la tradition du pragmatisme, mais qui est en voie d'être recouvert par des approches qui ont peut-être renoncé trop rapidement à la communauté que ce concept légitime? Cette réappropriation va à contre-courant du mouvement actuel de spécialisation, mais je crois qu'elle est seule

2. Ibidem, p. 61 .

3. John DeweY, Democracy and Education; An Introduction to the Philosophy of Education. New-York, The Free Press, 1966 ; première édition, London, Macmillan, 1916. 
porteuse de vie. «Ainsi pour les disciples d'Emmaüs: ils ne se persuadent de la présence divine que lorsque celle-ci les a quittés. » (Maurice Blanchot)

Département de philosophie

Université du Québec à Montréal 